\title{
Contribution anthropo-didactique à l'étude des effets de l'individualisme sur la création des inégalités scolaires
}

Cas des interactions verbales dans l'enseignement des mathématiques au cycle 3

Anthropo-didactical contribution to the study of the effects of individualism upon the creation of school inequalities. The case of verbal interactions in mathematical teaching at cycle 3

\section{Marie-Pierre Chopin et Bernard Sarrazy}

\section{(2) OpenEdition}

\section{Journals}

Édition électronique

URL : http://journals.openedition.org/educationdidactique/1919

DOI : 10.4000/educationdidactique.1919

ISSN : 2111-4838

Éditeur

Presses universitaires de Rennes

Édition imprimée

Date de publication : 12 décembre 2014

Pagination : 9-24

ISBN : 978-2-7535-39-66-2

ISSN : 1956-3485

Référence électronique

Marie-Pierre Chopin et Bernard Sarrazy, «Contribution anthropo-didactique à l'étude des effets de l'individualisme sur la création des inégalités scolaires », Éducation et didactique [En ligne], 8-2 | 2014, mis en ligne le 12 décembre 2016, consulté le 30 avril 2019. URL : http://journals.openedition.org/ educationdidactique/1919; DOI : 10.4000/educationdidactique.1919 


\title{
CONTRIBUTION ANTHROPO-DIDACTIQUE À L'ÉTUDE DES EFFETS DE L'INDIVIDUALISME SUR LA CRÉATION DES INÉGALITÉS SCOLAIRES

\author{
CAS DES INTERACTIONS VERBALES DANS L'ENSEIGNEMENT \\ DES MATHÉMATIQUES AU CYCLE 3
}

\author{
Marie-Pierre Chopin, université Bordeaux, Laboratoire Cultures Éducation Sociétés EA4140 \\ Bernard Sarrazy, université Bordeaux, Laboratoire Cultures Éducation Sociétés EA4140
}

\begin{abstract}
Larticle présente une recherche anthropo-didactique sur la contribution des interactions verbales maitre-élèves à l'avancée du temps didactique. Dans un premier temps, les auteurs examinent la fonctionnalité du caractère public ou privé des interactions afin de montrer leur rôle dans l'individualisation de l'enseignement et comme un instrument de gestion d'un collectif d'élèves. Dans un second temps, cette perspective est précisée : les auteurs s'intéressent à des interactions dont la visée est d'évacuer tout traitement didactique ou cognitif de certaines interventions d'élèves : les interactions « phatiques ». Les auteurs montrent leur forte utilité à l'avancée du temps didactique; ce résultat permet d'interroger l'idéologie individualiste fortement plébiscitée aujourd'hui
\end{abstract}

Mots-clés : interactions verbales, interactions phatiques, temps didactique, équité, efficacité.

Anthropo-didactical contribution to the study of the effects of individualism upon the creation of school inequalities The case of verbal interactions in mathematical teaching at cycle 3

The article presents an anthropo-didactic research about the role of verbal interactions into the advance of didactic time. In a first step, the authors examine the functionality of the public or private dimension of interactions in order to show their role into the individualization of teaching, and as a tool for the management of a pupils group. In a second time, they clarify this perspective. They study the interactions which aim is to expel didactic or cognitive treatment of pupils' interventions: the "phatic interactions". The authors show their utility for the advancing of didactic time. This result leads to question the individualist ideology, very acclaimed today

Keywords: Verbal interactions, phatic interactions, didactical time, equity, efficacy. 


\section{INTRODUCTION}

À la fin des années 80, l'école est fortement mais silencieusement marquée par l'idéologie libérale dont l'individualisme représente certainement la forme plus visible (Poupeau, 2003 ; Roiné, 2009). Le célèbre slogan «mettre l'enfant au centre du système éducatif », impulsé en 1989 par Jospin dans la définition de la Nouvelle politique pour l'école, en témoigne de façon emblématique. C'est à cette période également que la pédagogie différenciée - dont les effets pervers seront rapidement identifiés (Duru-Bellat, 1996 ; Mingat \& Duru-Bellat, 1997) - est « officialisée» (circulaire du 9/04/90). Les notions d'hétérogénéité, de diversité des élèves et des personnes, de groupes de besoins ou encore de dispositifs individualisés apparaissent aux plans officiels comme dans les idéologies professorales ${ }^{1}$. Elles restent encore aujourd'hui particulièrement présentes.

À côté des recherches précitées, celles menées par notre équipe dans les années 2000 ont permis de contribuer à la mise au jour de quelques effets de ces idéologies individualistes sur la création ou le maintien des inégalités scolaires en examinant leurs effets différenciateurs sur les acquisitions des élèves (Sarrazy, 2001, 2002 ; Chopin, 2007). La recherche présentée dans cet article permet de préciser et de mettre à l'épreuve les intuitions que ces travaux avaient fait émerger. La question à laquelle nous nous proposons de répondre en particulier concerne la manière dont les professeurs résolvent l'équation tripartite leur permettant de composer au mieux entre : a) la nécessité de faire progresser les connaissances pour le plus grand nombre d'élèves dans un temps nécessairement limité ; b) la diversité des niveaux initiaux des élèves dans la discipline enseignée (ici les mathématiques) ; c) la diversité (qualitative et quantitative) des modes de participation des élèves durant le temps d'enseignement. En d'autres termes, nous serons conduits ici à examiner la manière dont les professeurs font usage de l'un des instruments didactiques les plus puissants, à tout le moins les plus classiques ${ }^{2}$, pour faire avancer leur leçon: les interactions verbales. Ces dernières seront considérées dans leurs dimensions qualitatives (quelles modalités prennent-elles ?), pragmatiques (quelles fonctions assurent-elles ?) et surtout fréquentielles (sont-elles également distribuées selon les moments de la leçon, selon les types d'élèves ou selon les espaces interlocutoires où elles se manifestent - par exemple en « privé » ou en « public » ?).

L'article s'organisera ainsi en deux temps. Dans une première partie, nous resituerons plus en détail les questions présentées ci-dessus au sein de la perspective théorique (anthropo-didactique) qui les a vues naître, en proposant une synthèse des principaux résultats aboutissant à cette étude sur l'intérêt les interactions verbales prises comme révélatrices des idéologies professorales (quelles qu'en soient les origines), comme instruments didactiques des professeurs et enfin comme facteurs de déterminations des inégalités. Dans une seconde partie, nous présenterons le dispositif expérimental mis en place pour la réalisation de l'étude, les résultats obtenus et les analyses menées pour répondre à cet ensemble de questions que nous prendrons le risque de résumer ici d'une façon délibérément radicale : est-il toujours «bon » de répondre aux questions des élèves?

\section{PROBLÉMATIQUE : HÉTÉROGÉNÉITÉ DIDACTIQUE ET INTERACTIONS}

Toute action d'enseignement trouve sa raison d'être dans une hétérogénéité initiale entre les deux protagonistes du procès d'enseignement : le professeur (celui qui sait) et l'élève (celui qui ne sait pas, pas encore, ou qui croit savoir). La volonté de réduire cette distance initiale se manifeste par un ensemble d'actions et de dispositifs visant à faire naître ou à transformer les rapports des élèves à des situations dont la maîtrise nécessite les connaissances visées par l'enseignement ${ }^{3}$. Mais l'ensemble des rapports privés des élèves à ces objets d'enseignement est, de facto, hétérogène (sous l'effet de différences de trajectoires scolaires, d'intérêts, de niveaux scolaires, etc.). Pour pouvoir entreprendre ce travail de régulation, le travail du professeur consiste ainsi dans un premier temps à rendre manifeste et à optimiser l'hétérogénéité initiale des rapports que ses élèves entretiennent à la situation proposée.

Les travaux que nous avons menés conduisent ainsi à envisager ce phénomène comme une véritable création d'hétérogénéité, de la part du professeur, création constitutive de tout projet d'enseignement. En effet, c'est parce que la mise à jour des positionnements hétérogènes des élèves relativement à un objet de savoir dépend du type de situation proposée par le professeur (mettant en jeu sous telle ou telle 
forme un ensemble de connaissances) que l'action de « dévoilement » des différences de ces rapports apparaît toujours-déjà comme un produit de l'action didactique elle-même. Pour ne donner qu'un exemple trivial, songeons que le fait de proposer aux élèves des problèmes excessivement simples aura pour effet de maintenir le groupe dans une position d'homogénéité didactique (e.g. tous les élèves réussissent). À l'inverse, une leçon trop ambitieuse risquant d'être trop difficile pour une proportion importante d'élèves pourrait nuire à la création d'une hétérogénéité « satisfaisante " pour l'action didactique, c'està-dire permettant à l'enseignant de trouver une prise effective sur son groupe classe. En d'autres termes, quel que soit le niveau initial de la classe (élevé, faible...), le professeur doit nécessairement ajuster son action en fonction à la fois de son projet d'enseignement, des exigences définies par les programmes et des contraintes (en termes de niveau, de temps...) qui structurent son projet d'enseignement. En créant de l'hétérogénéité didactique, il inaugure paradoxalement la possibilité de sa réduction, par le jeu de son déplacement progressif ajusté à l'évolution de ses exigences didactiques. Les hétérogénéités initialement créées pourront, grâce à la mise en place de situations nouvelles, être réduites, puis recréées, selon des mouvements de succession ou d'enchâssement permettant leur déplacement. C'est ainsi, comme nous l'avons détaillé ailleurs (Chopin, 2010), qu'avancent les savoirs dans la classe, au rythme d'un temps non linéaire : le temps didactique.

Le modèle de l'hétérogénéisation didactique que nous venons d'exposer rapidement n'a pas été mis en défaut par les multiples observations réalisées concernant l'enseignement des mathématiques à l'école élémentaire et s'est même avéré d'une grande fécondité dans d'autres disciplines et à d'autres niveaux d'enseignement (par exemple, l'enseignement de la physique en classe de seconde, Chopin, 2012). Il a permis de renouveler l'approche des phénomènes d'enseignement en établissant que toute progression des connaissances se paie en gain d'hétérogénéité (Sarrazy, 2002), faisant de cette dernière une variable régulée par l'enseignement, et mettant en exergue l'intérêt d'étudier, dans une nouvelle perspective, l'un des instruments particulièrement important de ces régulations : les interactions maître-élèves ${ }^{4}$.

Un enjeu central de cette recherche est d'examiner le rôle des interactions en lien avec le milieu didactique mis en place par le professeur, et en tenant compte de la dimension proprement anthropologique du jeu interactif de la classe. Cette perspective s'inscrit ainsi dans le sillon des travaux menés par exemple par Voigt (1985), reprenant dans le champ didactique les apports ethnométhodologiques et interactionnistes de Garfinkel et Goffman. La particularité de notre étude est de centrer l'analyse sur une catégorie d'interactions particulières que nous désignerons, en référence à Roman Jakobson (1963), « interactions phatiques ${ }^{5}$ ». Leur fonction première est de permettre au professeur de maintenir l'élève dans le champ interlocutoire sans lui répondre effectivement sur le contenu même de son énoncé ; le professeur ne traite donc pas son intervention, ni cognitivement (pas de validation ou d'explications explicites), ni didactiquement (le professeur ne s'en sert pas pour exemplifier ce qu'il conviendrait de faire, de penser, de comprendre...). Dans la perspective du modèle d'hétérogénéisation didactique, nous faisons l'hypothèse que ce type d'interaction contribue à la régulation des hétérogénéités didactiques et donc à l'avancée du temps didactique

\section{PRÉSENTATION DE L'ÉTUdE}

\section{Le cadre général de la recherche}

L'étude présentée est extraite d'une recherche menée sur 8 classes de CM2 (soit 197 élèves), et sur 24 séances d'enseignement des mathématiques. Chaque séance observée, d'une heure, porte sur la résolution de problèmes dits "TTT», correspondant à la quatrième structure additive de la typologie élaborée par Vergnaud $(1990,1994)^{6}$. La particularité de cette structure est de ne mettre en jeu que des transformations positives ou négatives, sans qu'aucune indication ne soit fournie sur l'état numérique initial - d'où son appellation courante "TTT" " " $1^{\text {re }}$ Transformation - $2^{\mathrm{e}}$ Transformation Transformation composée "). L'énoncé ci-dessous présente un exemple-type de ce genre de problèmes :

Lou joue deux parties de billes.

Elle joue une partie. A la seconde partie, elle perd 4 billes.

Après les deux parties, elle a gagné 6 billes. Que s'est-il passé à la $1^{\text {re }}$ partie?

Cet objet d'enseignement est associé par Vergnaud à la question du passage de l'arithmétique 
à l'algèbre ${ }^{7}$, domaine d'étude important de la didactique des mathématiques (Barallobres, 2004 ; Broin, 2002 ; Carraher, Schliemann \& Brizuela, 2006, etc.). Il a été imposé à chaque professeur dans le cadre de notre dispositif expérimental.

Après avoir présenté le domaine mathématique concerné à chacun des 8 professeurs individuellement ainsi que les principales difficultés généralement rencontrées par les élèves de 10-11 ans sur ce thème, nous leur avons demandé d'organiser une séquence d'enseignement qui permettrait aux élèves de progresser dans la résolution de ce type de problème. Excepté le thème de la leçon, donc, le contenu même de la séquence (type d'exercices, etc.) était laissé complètement libre. Les professeurs avaient le choix d'utiliser ou non un assortiment de problème TTT que nous leur avions fourni, comme ils auraient pu le faire à partir de n'importe quel manuel.

En amont des séquences les élèves ont été soumis à un pré-test constitué de 22 problèmes TTT. Sur cette base, le niveau initial des 8 classes a été mesuré et les 197 élèves ont été répartis en trois groupes ( « bon », « moyen », « faible »), en fonction de leur score $^{8}$. Aucune classes ne différait du point de vue de la distribution des niveaux scolaires $(\chi 2=15,18$; p. = .37). À l'issue des séquences, un post-test (identique au pré-test) a permis de mesurer les progressions des élèves grâce à un indice de progression Ip dont le mode d'élaboration est présenté en annexe 3).

\section{Étude des interactions}

Concernant l'étude plus particulière des interactions, l'utilisation de la vidéo a permis l'élaboration d'une grille d'analyse détaillée du champ interactif. En tout, 12 modalités interactives ont été définies : 8 de type didactique, c'est-à-dire concernant directement le savoir en jeu (il s'agit pour l'essentiel de questions posées par l'enseignement à propos de la solution à un problème, d'une information délivrée, etc. $)^{9}$, et 4 de type proto-didactique, c'est-à-dire entourant et accompagnant le jeu didactique ${ }^{10}$.

\section{Distinction public/privé}

Ces 12 modalités ont ensuite été dédoublées selon un critère qui nous intéressera particulière- ment pour l'élaboration des premiers résultats de l'étude : la distinction entre interactions publiques (le professeur échange avec un ou plusieurs élèves tout en étant audible de l'ensemble de la classe) et interactions privées (menées spécifiquement auprès de certains élèves). Dans ce dernier cas (interactions privées), notons que l'utilisation d'un micro-cravate porté par le professeur a été essentielle pour nous permettre de retranscrire finement les échanges de la séquence.

\section{Interactions phatiques}

Comment avons-nous caractérisé les interactions phatiques dans cet ensemble d'interactions?

Ces dernières font partie des interactions dites « didactiques»; elles sont produites à l'occasion d'un échange autour du savoir en jeu, lorsque le professeur doit gérer l'intervention d'un élève dans le déroulement de la leçon. Trois cas peuvent être considérés :

1. Le professeur a posé une question à l'ensemble de la classe et un élève propose une réponse qui ne correspond pas à ses attentes ; 2 . Le professeur a interrogé un élève nommément et ce dernier propose une réponse non satisfaisante $\mathrm{ll}^{11}$;

3. Un élève intervient de manière spontanée au cours de la leçon pour proposer une réponse, une manière de procéder, etc., qui n'est pas utile pour le professeur.

Dans ces trois cas, le professeur se trouve face à l'alternative suivante :

- Produire un traitement cognitif ou didactique de l'intervention de l'élève (soit en poursuivant l'échange interactif par des régulations, soit en validant ou en invalidant sa proposition lui conférant ainsi un statut didactique utile au reste de la classe) ;

- Produire un traitement phatique de l'intervention, c'est-à-dire l'accueillir sans pour autant réaliser de régulation cognitive ou de traitement didactique.

Parmi les traitements phatiques, on trouvera par exemple des réponses du professeur du type « Oui, oui, c'est très intéressant mais on verra ça plus tard ", "Peut-être... Qui propose autre chose ? », etc. Mais notons que dans de nombreux cas, la dimension phatique d'une interaction n'apparaît pas littéra- 
lement, au sens où certains termes ou expressions pourraient l"'encapsuler". Dans la lignée des travaux bien connus d'Austin (1970) puis de Searle (1982), on soulignera que la fonction phatique d'une interaction relève généralement du discours de seconde intention (dont Searle proposait d'ailleurs d'entreprendre la phénoménologie, attestant par-là l'idée selon laquelle la fonction de l'expression dépendait avant tout de la manière dont elle nous apparaissait). Ainsi, au-delà des quelques formes littérales stables identifiées dans notre corpus ( "Oui, oui...", « C'est ça mais on verra plus tard », etc.), ce sont bien dans les circonstances (définies par les trois cas rappelés ci-dessus) plus que dans les termes utilisés, qu'il faut rechercher les causes de l'affectation de telle interaction à la catégorie des interactions phatiques

\section{RÉSULTATS ET ANALYSES}

\section{Résultats 1 : Interactions publiques ou privées - logiques anthropologiques/fonctionnalité didactique}

Les premiers résultats établis portent sur la répartition des interactions maître-élève(s) entre la sphère publique (face à l'ensemble de la classe) et l'espace privé (entre le professeur et un élève ou un petit groupe d'élèves en particulier). Malgré des styles interactifs variables entre les 8 enseignants de l'échantillon (certains présentent des volumes globaux d'interaction deux fois plus importants que d'autres), on note une corrélation positive très nette entre le nombre d'interactions publiques et privées pour chaque classe de l'étude. (Figure 1)

Notons que cette corrélation se maintient pour chacun des niveaux scolaires (élèves bons, moyens ou faibles) ${ }^{12}$. Ce résultat, montrant l'indépendance entre, d'une part, la régularité de la répartition entre interactions publiques et privées et, d'autre part, le volume total d'interaction pour chaque professeur, indique donc que ce type de répartition public/privé est un effet structurel dans le processus d'enseignement. Les résultats suivants permettent d'avancer quelques hypothèses pour expliquer l'origine d'une telle régularité dans l'économie didactique de professeurs.

Le modèle de l'hétérogénéisation didactique permet de penser que la fonctionnalité des interactions publiques diffère très fortement de celles opérant dans l'espace privé. Ce qu'énonce le professeur dans l'espace public, par l'intermédiaire d'un échange avec un élève, participerait à l'aménagement d'un milieu censé permettre le déplacement des hétérogénéités didactiques révélées dans l'enseignement. Une telle hypothèse est étayée par les résultats suivants, portant sur la manière dont les professeurs

Figure 1 - Corrélation entre le nombre d'interactions publiques et le nombre d'interactions privées pour chaque niveau scolaire dans les 8 classes

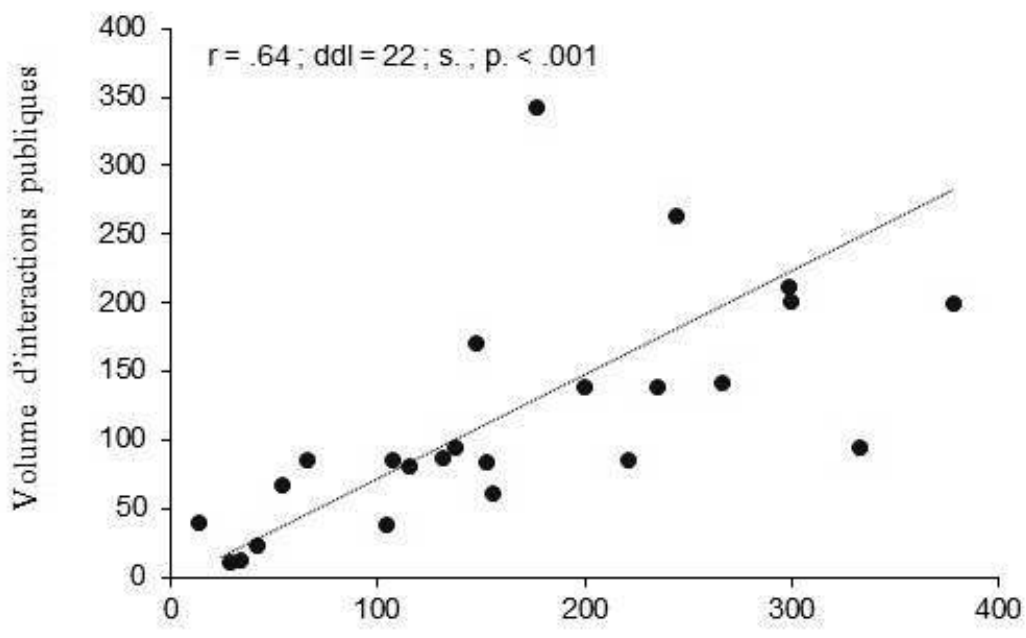

Volume d'interactions privées 
traitent les réponses erronées données par leurs élèves au cours de l'enseignement.

Lorsque l'on se place dans l'espace public, les résultats montrent en effet des corrélations négatives entre la progression des élèves forts $(p<.20)$ et moyens $(\mathrm{p}<.05)$ et le traitement des réponses fausses données par les élèves faibles. Pour le dire autrement, les élèves forts et moyens progressent d'autant moins que les erreurs des élèves faibles sont traitées dans l'espace public. Ce résultat ne se maintient pas dans l'espace privé où le traitement que fait le professeur des réponses erronées des élèves faibles n'influe en rien sur les progressions des autres élèves.

Concernant les élèves faibles maintenant, dont on pourrait penser qu'ils sont les bénéficiaires du phénomène précédent (l'intérêt spécifique porté par le professeur au traitement de leurs réponses fausses leur serait favorable), on ne note pourtant aucune corrélation significative entre le traitement que fait le professeur de leurs réponses erronées et leur progression entre le pré-test et le post-test. Chose particulièrement surprenante, un tel résultat cette fois-ci se maintient dans la sphère privée.

Que conclure de ces résultats?

Que la fonctionnalité des interactions maîtreélève(s) doit être comprise au sein de l'économie générale de l'avancée du savoir dans la classe, et non sous l'aspect de leur effet direct sur la compréhension de tel ou tel élève initiateur d'une question ou proposant une réponse à une interrogation du professeur. Ainsi, les résultats montrent que le traitement des erreurs des élèves faibles nuit à l'économie générale de l'avancée du temps didactique. Plusieurs explications apparaissent :
$1 /$ celle du caractère chronophage de ces actions de traitement des réponses fausses données par les élèves faibles ;

2/ celle des effets de brouillage possiblement induits sur les élèves moyens et forts par le traitement public des erreurs des élèves faibles.

En d'autres termes, alors que les interactions apparaissent généralement - sinon dans la littérature de recherche, tout au moins pour le sens commun pédagogique - comme le moyen privilégié de l'individualisation de l'enseignement, elles se révèlent avant tout dans la pratique un instrument de gestion d'un collectif d'élèves.

Dans ce qui suit, nous proposons de mettre à l'épreuve une telle hypothèse en nous intéressant à un ensemble plus large d'interactions spécialement caractérisées par le fait d'évacuer tout traitement didactique ou cognitif des interventions des élèves: les interactions phatiques.

\section{Résultat 2 : La distribution des interactions phatiques selon le niveau scolaire}

Les résultats font apparaître une relation fortement significative entre les progrès réalisés par les élèves et le volume d'interactions phatiques. Dit autrement, plus la proportion des interactions phatiques est élevée (rapportée aux autres types d'interaction pour un même professeur) plus le niveau moyen des élèves s'élève $(r=.84 ; \mathrm{s}$. ; $\mathrm{p} .<.01)$. C'est ce que résume la figure suivante :

Figure 2 - Corrélation entre la proportion d'interactions phatiques et la progression des élèves

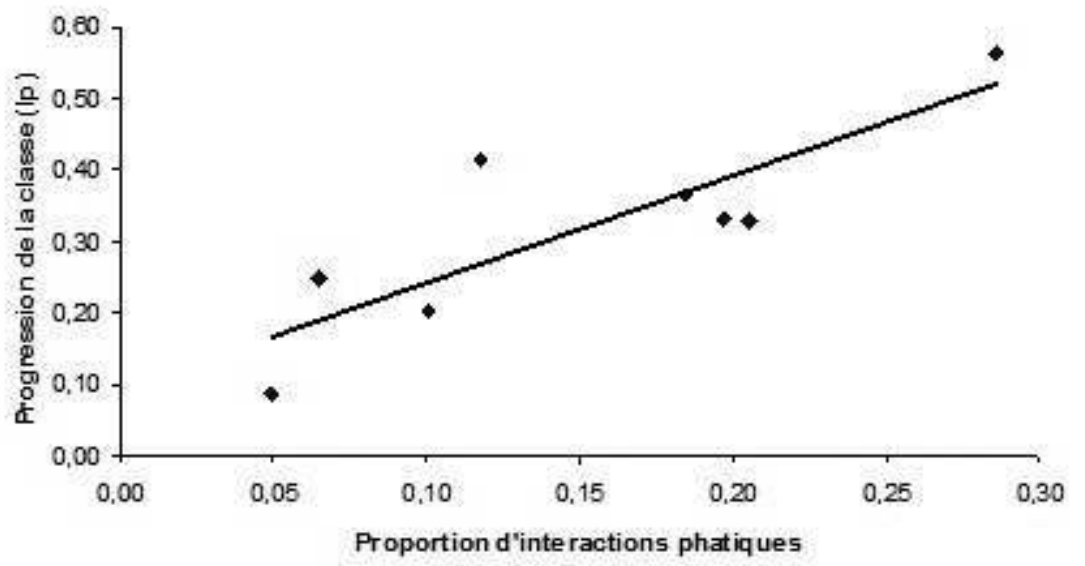


En complément de ce résultat, notons que cet effet se manifeste très fortement et très significativement sur les élèves moyens $(r=.87)$ et moins nettement sur les élèves faibles $(r=.58)$ et pas du tout sur les bons élèves. On voit donc bien ici une manifestation des contraintes chronogénétiques qui pèsent sur le professeur indépendamment de son style d'enseignement (caractérisable par la variabilité très forte entre les volumes d'interactions et leur distribution qualitative).

Quelles fonctions assurent ces interactions particulières? La réponse que nous soutiendrons est celle de la régulation du temps didactique : ce ne sont pas les interactions phatiques qui font avancer mécaniquement le temps didactique mais bien leur fonction qualitativement distribuée dans le jeu interactif. Le tableau 1 ci-dessous résume les informations permettant d'examiner la relation entre:

- d'un côté, la progression réalisée par les élèves de chaque classe (indice Ip, deuxième colonne du tableau) - plus l'indice est élevé, plus les classes ont progressé ;

- de l'autre, le seuil de signification des Chideux (troisième colonne du tableau) permettant d'examiner si la distribution des interactions phatiques dans chaque classe est significativement différenciée selon le niveau scolaire des élèves - plus le seuil de signification est faible, plus les interactions phatiques se distribuent de façon différenciée, en fonction du niveau scolaire des élèves auxquels elles sont adressées (c'està-dire que l'enseignant n'utilise pas les interactions phatiques de la même manière avec les bons élèves, les moyens et les faibles).

Les trois dernières colonnes du tableau 1 font apparaître les attractions et les répulsions (le sens des écarts à l'indépendance). Elles permettent d'apprécier quel type d'élève est concerné par les interactions phatiques du professeur (les bons élèves, les élèves moyen ou les faibles). (Tableau 1)

Comme on peut le voir ci-dessous, les classes qui progressent le plus sont celles où la distribution des interactions phatiques est significativement différenciée selon le niveau scolaire. Réciproquement, ce sont celles dont les traitements phatiques ne sont pas différenciés qui enregistrent les progressions les plus faibles $(r=-.73 ; \mathrm{s} . ; \mathrm{p} .<.05)$. Une analyse plus qualitative des trois dernières colonnes montre enfin que, lorsqu'il y a un usage différencié des interactions phatiques par le professeur en fonction des niveaux scolaires de ses élèves (seuil de signification du Chi-deux inférieur à .05), ce sont les élèves moyens et les faibles qui sont les destinataires principaux des interactions phatiques. Il y a ainsi de bonnes raisons d'affirmer que le professeur parvient, par ce jeu interactif, tout à la fois à maintenir le canal de communication avec ces élèves-là sans ralentir l'avancée du temps didactique pour l'ensemble de la classe.

\section{Résultat 3 : Taux d'interactions phatiques et visibilité didactique}

Un troisième faisceau de résultats permet pour finir de reconnecter les éléments mis en évidence ci-dessus avec la problématique plus globale de l'individualisation de l'enseignement et de ses effets.

La recherche complète dont sont issus les résultats présentés avait permis de mettre en évidence quels agencements de conditions, dans l'aménagement des situations d'enseignement proposées par les professeurs, expliquaient statistiquement les

Tableau 1 - Progression des classes et distribution des interactions phatiques

\begin{tabular}{|c|c|c|c|c|c|}
\hline \multirow[t]{2}{*}{ Classe } & \multirow{2}{*}{$\begin{array}{l}\text { Progression } \\
\text { (Ip) }\end{array}$} & \multirow{2}{*}{$\begin{array}{l}\text { Seuil de signification } \\
\text { du Chi-deux }\end{array}$} & \multicolumn{3}{|c|}{ Attractions / répulsions des interactions phatiques } \\
\hline & & & Élèves bons & Élèves moyens & Élèves faibles \\
\hline Ecol & 0,33 & .21 & + & - & + \\
\hline Eco2 & 0,56 & .00 & - & - & + \\
\hline Eco3 & 0,41 & .01 & - & + & - \\
\hline Eco4 & 0,33 & .05 & - & - & + \\
\hline Eco5 & 0,09 & .52 & - & + & + \\
\hline Eco6 & 0,20 & .97 & - & - & + \\
\hline Eco 7 & 0,25 & .69 & + & + & - \\
\hline Eco8 & 0,36 & .03 & - & + & - \\
\hline
\end{tabular}


progressions réalisées par les élèves au cours des enseignements dispensés. En étudiant de près la nature des assortiments didactiques proposés aux élèves (Esmenjaud-Genestoux, 2002), les types de contrats en vigueur dans la classe (Brousseau, 1988, 1966), et la nature des régulations proposées par les professeurs, la recherche avait permis de mettre en évidence le rôle primordial d'une variable pour l'avancée du temps didactique dans la classe : la visibilité didactique.

Cette visibilité - nous renvoyons le lecteur à la présentation plus complète de ce concept dans Chopin, 2008) - correspond à la possibilité donnée au professeur de voir et de contrôler les phénomènes d'hétérogénéisation didactique au cours de l'enseignement. En particulier, cette visibilité repose sur la possibilité qu'a le professeur d'interpréter les comportements de ses élèves au cours de l'enseignement en relation avec la nature des situations didactiques proposées et, plus fondamentalement, avec leur structuration proprement épistémique : quel type de connaissance est requise par cette situation et est à l'origine de l'affectation de tel ou tel élève à telle position particulière (il ne sait pas faire, il fait de telle façon, non conforme à celle que je cherche à lui enseigner, etc.) ?

L'étude a permis de montrer que la visibilité didactique expliquait fortement, et même prioritairement (avant le niveau scolaire initial de l'élève par exemple ${ }^{13}$ ), les progressions réalisées : plus la visibilité didactique est importante, plus les élèves progressent, quel que soit leur niveau. Lorsque la visibilité est réduite, alors c'est le niveau scolaire de l'élève qui explique statistiquement la progression de l'élève.

Pour en revenir à l'examen des interactions phatiques, nos résultats ont permis de montrer que les professeurs présentant un taux très faible d'interactions phatiques sont aussi ceux caractérisés par une visibilité didactique faible (la possibilité qu'ils appréhendent précisément l'état d'hétérogénéisation didactique de leur classe est réduite). En d'autres termes, plus la visibilité didactique est importante, plus les professeurs enregistrent une proportion importante d'interactions phatiques dans leur discours. À l'issue d'une telle étude, ce résultat ne saurait surprendre : c'est bien la possibilité qu'a le professeur de voir et de contrôler les phénomènes d'hétérogénéisation de sa classe qui lui permettra de sélectionner, sur le vif et sans nécessaire prise de conscience, les interactions les plus propices à l'économie globale de l'avancée du savoir dans la classe. À l'inverse, tout se passe comme si les professeurs présentant une visibilité didactique réduite appréhendaient au même niveau l'ensemble des interventions des élèves, sans traitement différencié - bien que didactiquement efficient - de ces dernières.

Ainsi, plus un professeur individualise son enseignement (par souci de venir en aide aux plus faibles), plus il perd le contrôle des phénomènes d'hétérogénéisation pourtant essentiels à la progression globale de la classe, y compris celle des plus faibles.

\section{CONCLUSION}

Si l'on devait conclure de façon aussi lapidaire que nous avions résumé notre question initiale on pourrait dire qu'il n'est pas toujours " bon » de répondre effectivement aux élèves, particulièrement pour les élèves moyens et faibles. Individualiser l'enseignement est pourtant souvent présenté comme un moyen de réduire les difficultés des élèves. Les directives ministérielles adressées aux professeurs vont pourtant clairement dans ce sens. Voici ce qu'on pouvait lire par exemple dans les programmes pour l'enseignement primaire de 2008 :

Tableau 2 - Analyse de variance à un facteur de classification : les progressions des élèves (Ip) sont-elles liées à la visibilité didactique (VD) ?

\begin{tabular}{lllc}
\hline & Somme des carrés & Nombre & Variance \\
Sources & des écarts à & De degrés & De liberté \\
de variation & la moyenne & 2 & 1,25 \\
\hline Effets des traitements & 2,51 & 173 & 0,19 \\
A l'intérieur des groupes & 32,43 & & $\mathrm{p}=.002$ \\
Variation totale & 34,94 & & \\
\hline
\end{tabular}


«Les élèves en difficulté doivent pouvoir bénéficier d'une aide personnalisée et différenciée dès que les premières difficultés apparaissent et avant qu'elles ne soient durablement installées. [...] Le rôle de l'enseignant est [...] d'aider ses élèves à progresser dans la maîtrise des objectifs fixés par les programmes [...] il lui revient de choisir les méthodes les plus adaptées aux caractéristiques individuelles et aux besoins spécifiques de ses élèves. »(MEN. PROG, 2008, 10-11).

Si beaucoup d'enseignants adhèrent à l'idée de ce projet ambitieux de faire progresser chaque élève, et notamment ceux " en difficulté », on leur reproche par ailleurs de ne pas consacrer suffisamment de place à cette individualisation. Comprendre les raisons de ce hiatus était une des visées de cette étude. On l'a vu, les interactions sont sans conteste un moyen privilégié d'individualiser l'enseignement et les échanges verbaux avec leurs élèves semblent contribuer à déceler leurs difficultés et si possible les réguler. C'est pour répondre à un tel impératif que certains d'entre eux, notamment les plus jeunes dans le métier, s'engagent dans des échanges interactifs souvent longs et coûteux avec leurs élèves, enchaînant demandes d'explicitation, reprises et reformulations, tentant ainsi de traiter les erreurs des élèves (notamment des plus faibles) dès leur apparition. Nos résultats permettent d'en pointer les limites et même les écueils.

Quel que soit le niveau scolaire des élèves, la progression des connaissances apparaît ici clairement favorisée par l'usage des interactions phatiques, c'està-dire celles qui écartent ou diffèrent les traitements effectifs de leurs interventions. "Laissez-moi faire mon métier, vous comprendrez plus tard », telle pourrait être la justification didactique des interactions phatiques. Plus précisément, c'est lorsque ces interactions concernent spécifiquement les élèves moyens et faibles (c'est-à-dire ceux qui sont censés être les principaux bénéficiaires d'un enseignement individualisé), que les progressions sont les plus importantes.

Bien évidemment, il convient de rappeler au moment de clore cet article que les interactions phatiques ne doivent pas être considérées comme efficaces en soi, mais bien comme un indice (et même un effet) de la visibilité didactique du professeur dans le cours de sa leçon. La perspective anthropodidactique adoptée dans cet article a en effet permis de comprendre leur usage comme la résultante d'un double assujettissement : au temps didactique d'une part (faire avancer le savoir pour le plus grand nombre d'élèves dans le temps qui lui est donné) ; à un impératif pédagogique et éthique d'autre part : ne pas laisser des élèves "sur le côté ». C'est bien dans ce cadre que la fonction d'une interaction phatique devient essentielle, c'est-à-dire en tant que régulateur du jeu didactique : un échange trop long avec un élève met en péril l'avancée de la leçon pour le reste de la classe et même souvent peut être générateur de chahut. Autrement dit, l'efficacité et l'équité de l'enseignement trouvent leurs conditions de possibilité dans l'existence, au sein du jeu interactif, d'une proportion raisonnable de traitements phatiques, ceux dont on pourrait penser a priori qu'ils sont dénués de toute propriété didactique et qui pourtant sont tout à fait fondamentaux pour concilier ces deux impératifs (didactiques et pédagogiques) trop souvent envisagés comme contradictoires. 


\section{RÉFÉRENCES}

Altet Marguerite (1994) Comment interagissent enseignant et élèves en classe ?, Note de synthèse, Revue Française de Pédagogie, n 107, Lyon, INRP, 123-139.

Austin J.-L. (1970) Quand dire, c'est faire. sl : Éditions du Seuil, 202 p., coll. "Points. Essais".

Barallobres Gustavo (2004) La validation intellectuelle dans l'enseignement introductif de l'algèbre, Recherches en Didactique des Mathématiques, vol. 24, $n^{\circ}$ 3, Grenoble, La Pensée Sauvage, 285-328.

Bayer Édouard (1973) Lanalyse des processus d'enseignement, Revue Française de Pédagogie, n 24 , Lyon, INRP, 30-40.

Bayer Édouard et al. (1979) Essai d'analyse de la participation des élèves en classe hétérogène, Revue Française de Pédagogie, n 49, Lyon, INRP, 45-61.

Bressoux Pascal (1990) Méthodes pédagogiques et interactions verbales dans la classe : quel impact sur les élèves de CP ?, Revue Française de Pédagogie, n 93, Lyon, INRP, 17-26.

Bressoux Pascal, Bru Marc, Altet Marguerite, LecomteLambert Claire (1999) Diversité des pratiques d'enseignement à l'école élémentaire, Revue Française de Pédagogie, $\mathrm{n}^{\circ}$ 126, Lyon, INRP, 97-110.

Broin Dominique (2002) Arithmétique et Algèbre élémentaires scolaires, Thèse de doctorat en didactique des mathématiques, université Bordeaux 1, 2002.

Brousseau Guy (1988) Le contrat didactique : le milieu, Recherches en Didactique des Mathématiques, vol. 9, $n^{\circ}$ 3, Grenoble, La Pensée Sauvage, 309-336.

Brousseau Guy (1996) Théorie des situations didactiques, Cours donné à l'Université de Montréal en juin 1997 à l'occasion de la remise du doctorat honoris causa à G. Brousseau.

Brousseau Guy (1998) Théorie des situations didactiques, Grenoble, La Pensée Sauvage.

Carraher David W., Schliemann Analúcia D., Brizuela Barbara M. (2006) Arithmetic and Algebra in Eraly Mathematics Education, Journal for Research in Mathematics Education, vol. 37, $\mathrm{n}^{\circ} 2$, s.l., National Council of Teachers of Mathematics, 87-115.

Chopin Marie-Pierre (2007) Le temps didactique dans l'enseignement des mathématiques, Approche des modes de régulation des hétérogénéités didactiques, Thèse de doctorat, université Victor Segalen, Bordeaux, novembre 2007.

Chopin Marie-Pierre (2008) Les hétérogénéités : quels critères, quelles fonctions ?, dans Rouchier André et al. (éds), Actes de la XIII école d'été de Didactique des Mathématiques (Sainte-Livrade, Lot-et-Garonne, du 18 au 26 août 2005), Grenoble : la Pensée Sauvage, Cédérom d'accompagnement.

Chopin Marie-Pierre (2010) Le temps didactique et ses niveaux d'étude : enjeux d'une clarification conceptuelle pour l'analyse des pratiques d'enseignement, Recherches en didactique des mathématiques, $\mathrm{n}^{\circ} 30(1)$, Grenoble, La Pensée Sauvage, 83-112.
Chopin Marie-Pierre (2012) Reprendre la leçon, renouer le temps didactique : spiralité du temps du savoir et ajustement contractuel de début de séance en physique seconde, Éducation et didactique, vol. 6, n 3, Rennes, PUR, 21-40.

Crahay Marcel (1989) Contraintes de situation et interaction maître-élèves : changer se façon d'enseigner, Revue Française de Pédagogie, n 88, Lyon, INRP, 67-94.

Duru-Bellat Marie (1996) De quelques effets pervers des pédagogies différenciés, Éducations, n ${ }^{\circ} 7$, s.l., 12-15.

Esmenjaud-Genestoux Florence (2002) Les assortiments didactiques, dans Dorier Jean-Luc, Artaud Michèle, Artigue Michelle, Berthelot René \& Floris Ruhal (Éds), Actes de la $11^{\mathrm{e} e ́ c o l e ~ d ' e ́ t e ́ ~ d e ~ d i d a c t i q u e ~ d e s ~ m a t h e ́-~}$ matiques, Grenoble, La Pensée Sauvage, (version électronique)

Felouzis Georges (1993) Interactions en classe et réussite scolaire, Une analyse des différences filles-garçons, Revue Française de Sociologie, $\mathrm{n}^{\circ} 34$, Paris, Presses de Sciences Po, 199-222.

Filliettaz Laurent, Schubauer-Leoni Maria-Luisa, (dir.) (2008) Processus interactionnels et situations éducatives, Bruxelles, De Boeck.

Flanders Ned A. (1966) Interaction analysis in the classroom: A manual for observers, Univ. of Michigan, Michigan School of Education.

Giroux Jacinthe, Rene de Cotret Sophie (2003) Le temps didactique dans trois classes de secondaire I (doubleurs, ordinaire, forts), Éducation et francophonie, $\mathrm{n}^{\circ} 2$ XXXI,Ottawa, Association Canadienne d'éducation de langue française, (revue en ligne).

Guernier Marie-Cécile, Durand-Guerrier Vivianne, Sautot Jean-Pierre (Dir.) (2007) Interactions verbales, didactiques et apprentissages : recueil, traitement et interprétation didactiques des données langagières en contextes scolaires, Besançon, Presses Universitaires de FrancheComté.

Jakobson Roman (1963) Essais de linguistique générale, 1. Les fondations du langage, Paris, Minuit.

Marchive Alain (2006) Approche anthropo-didactique des phénomènes d'enseignement et de formation : contribution à l'étude des rapports entre pédagogie et enseignement, Note de synthèse pour l'habilitation à diriger des recherches, université de Bordeaux 2, Bordeaux, 2006.

MC Dermott Ray (1976) Kids make sense: An ethnographic account of the interactional manaqement of success and failure in one first-grade classroom, Unpublished Ph. D. Standtord University, Anthropology Department.

Mingat Alain, Duru-Bellat Marie (1997) La constitution de classes de niveau dans les collèges: les effets pervers d'une pratique à visée égalisatrice, Revue Française de sociologie, $\mathrm{n}^{\circ} 43,8$, Paris, Presses de Sciences Po, 754789.

Ministère de l'Éducation nationale (2008) Préambule des programmes de l'école primaire, (Arrêté du 9 juin 2008), Bulletin officiel hors-série $\mathrm{n}^{\circ} 3$ du 19 juin 2008.

Postic Marcel (1971) Lanalyse des actes pédagogiques des 
professeurs de sciences, Les Sciences de l'Éducation pour l'Ère Nouvelle, $\mathrm{n}^{\circ} 1$, Caen, Centre d'études et de recherche en sciences de l'éducation, 57-119.

Poupeau Franck (2003) Une sociologie d'état : Lécole et ses experts en France, Paris, Éditions Raisons d'Agir.

Roine Christophe (2009) Cécité didactique et discours noosphériens dans les pratiques d'enseignement en SEGPA: une contribution à la question des inégalités, Thèse de doctorat, université Victor Segalen Bordeaux 2, Bordeaux, Novembre 2009.

Sarrazy Bernard (1996) La sensibilité au contrat didactique, Rôle des Arrières-plans dans la résolution de problèmes d'arithmétique au cycle trois, Thèse de doctorat, université Victor Segalen Bordeaux 2, Bordeaux, janvier 1996.

Sarrazy Bernard (2001) Les interactions maître-élèves dans l'enseignement des mathématiques: Contribution à une approche anthropo-didactique des phénomènes d'enseignement, Revue Française de Pédagogie, n ${ }^{\circ}$ 136, Lyon, INRP, 117-132.

Sarrazy Bernard (2002) Les hétérogénéités dans l'enseignement des mathématiques, Educational Studies in Mathematics, $\mathrm{n}^{\circ} 49$, S.L., Springer, 89-117.

Searle J. R. (1982). Sens et expression : étude de théorie des actes de langage. Paris : éd. De minuit, 243 p., coll. «Le sens commun ».

Vergnaud Gérard (1990) La théorie des champs conceptuels, Recherches en didactique des mathématiques, $\mathrm{n}^{\circ} 10$ (23), Grenoble, La Pensée Sauvage, 133-170.

Vergnaud Gérard (1994) L'enfant, la mathématique et la réalité : Problèmes de l'enseignement des mathématiques à l'école élémentaire, Berne, Peter Lang.

Voigt Jürgen (1985) Patterns and routines in classroom interaction, Recherches en Didactique des Mathématiques, $n^{\circ}$ 6, 1, Grenoble, La Pensée Sauvage, 69-118. 
ANNEXE 1 :

\section{CHAMP CONCEPTUEL DES STRUCTURES ADDITIVES (VERGNAUD, 1994 [1981])}

La Théorie des Champs Conceptuels (Vergnaud, 1990a) entend fournir un cadre aux recherches sur les activités cognitives complexes, principalement sur les apprentissages scientifiques et techniques. Vergnaud distingue trois fonctions à cette théorie :

repérer et étudier les filiations et les ruptures entre connaissances du point de vue de leur contenu conceptuel ; analyser la relation entre les concepts (connaissances explicites) et les invariants opératoires (connaissances implicites) qui apparaissent dans les conduites des sujets en situation ;

analyser les relations entre signifiants et signifiés.

La classification des structures additives et soustractives en six catégories répond à la première de ces fonctions. Elle s'organise selon la nature des nombres en jeu dans les opérations basiques (nombres « mesure », que Vergnaud symbolise par un carré, nombres « transformation » ou « état relatif » symbolisés par un cercle) et selon le type de relation entretenue par ces nombres (composition, application de transformation, comparaison). La figure suivante résume ces six catégories:

Fig. a - Les structures additives d'après Vergnaud (1989, p. 218)

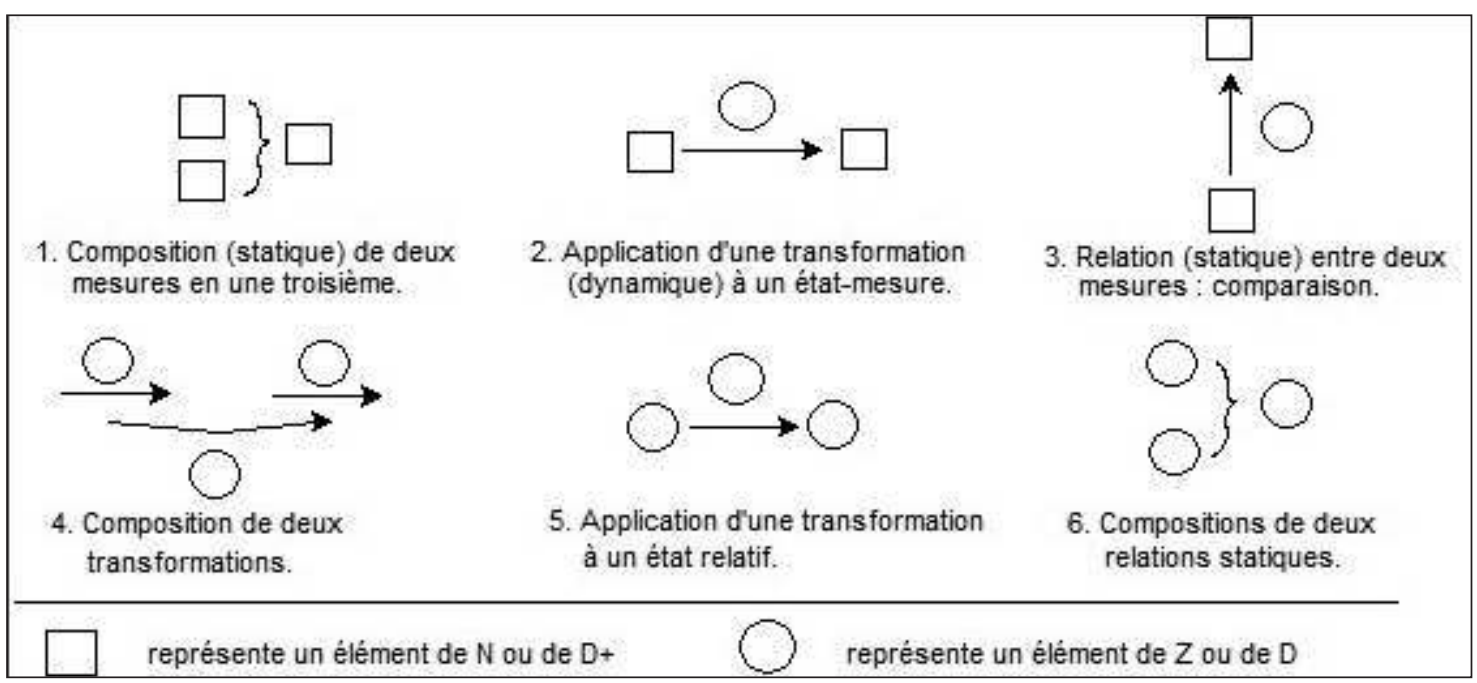

ANNEXE 2 :

\section{DÉFINITION DU NIVEAU SCOLAIRE DES ÉLÈVES}

Le pré-test offre la possibilité de mesurer le niveau initial des élèves de l'échantillon. Chacun d'entre eux y obtient un score sur 22 points sur la base duquel nous opérons un codage en classes ${ }^{14}$. De cette façon, nous affectons un niveau scolaire (NS) à chaque élève :

- de 0 à 7 problème(s) réussi(s) au pré-test : niveau « faible»;

- de 8 à 14 problèmes réussis au pré-test : niveau « moyen»;

- de 15 à 22 problèmes réussis au pré-test : niveau « bon ».

Pour faciliter l'écriture, nous nous passerons désormais des guillemets pour identifier les catégories d'élèves. Nous parlerons d'élèves faibles, moyens, ou forts en référence à cette construction initiale.

Le tableau suivant présente la distribution des effectifs de chaque classe du point de vue du niveau scolaire : 
Tab. a -Distribution des effectifs selon le niveau scolaire (NS) pour les huit classes

\begin{tabular}{|c|c|c|c|c|}
\hline & \multicolumn{3}{|c|}{ Niveau scolaire (NS) } & \multirow[b]{2}{*}{ Total } \\
\hline & Bon & Moyen & Faible & \\
\hline \multirow[t]{2}{*}{ Ecol } & 8 & 9 & 8 & 25 \\
\hline & $32 \%$ & $36 \%$ & $32 \%$ & \\
\hline \multirow[t]{2}{*}{ Eco2 } & 4 & 13 & 4 & 21 \\
\hline & $19 \%$ & $62 \%$ & $19 \%$ & \\
\hline \multirow[t]{2}{*}{$E \operatorname{co3}$} & 5 & 14 & 4 & 23 \\
\hline & $22 \%$ & $61 \%$ & $17 \%$ & \\
\hline \multirow[t]{2}{*}{ Eco4 } & 9 & 12 & 7 & 28 \\
\hline & $32 \%$ & $43 \%$ & $25 \%$ & \\
\hline \multirow[t]{2}{*}{ Eco5 } & 6 & 11 & 12 & 29 \\
\hline & $21 \%$ & $38 \%$ & $41 \%$ & \\
\hline \multirow[t]{2}{*}{ Eco6 } & 10 & 14 & 3 & 27 \\
\hline & $37 \%$ & $52 \%$ & $11 \%$ & \\
\hline \multirow[t]{2}{*}{ Eco7 } & 3 & 10 & 5 & 18 \\
\hline & $17 \%$ & $56 \%$ & $28 \%$ & \\
\hline \multirow[t]{2}{*}{ Eco8 } & 6 & 12 & 8 & 26 \\
\hline & $23 \%$ & $46 \%$ & $31 \%$ & \\
\hline \multirow[t]{2}{*}{ Total } & 51 & 95 & 51 & 197 \\
\hline & $26 \%$ & $48 \%$ & $26 \%$ & \\
\hline
\end{tabular}

Notons que le classement du niveau scolaire ainsi défini concorde avec la manière dont les professeurs évaluent leurs élèves en mathématiques ${ }^{15}\left(\chi^{2}=73.25\right.$; s. ; $\left.\mathrm{p}=.001\right)$.

Comparaison du niveau initial des huit classes

D’après le tri croisé présenté dans le Tab a - ci-dessus, les huit classes de l'échantillon ne diffèrent pas du point de vue du niveau des élèves $\left(\chi^{2}=23.68\right.$; n.s. ; $\left.\mathrm{p}=.37\right)$. Une analyse de variance à un facteur de classification confirme ce résultat à partir des distributions des scores des élèves au pré-test :

Ces huit classes ne diffèrent donc pas de manière significative du point de vue de leur réussite au pré-test (et donc de leur niveau initial).

Tab. b -Analyse de variance à un facteur de classification : le niveau initial des élèves (score au pré-test) varie-t-il selon la classe $(\mathrm{ECO})^{16}$ ?

\begin{tabular}{lllll}
\hline Sources & $\begin{array}{l}\text { Somme des carrés } \\
\text { des écarts à }\end{array}$ & $\begin{array}{l}\text { Nombre } \\
\text { de degrés } \\
\text { de moyenne }\end{array}$ & $\begin{array}{l}\text { Variance } \\
\text { dearré moyenen })\end{array}$ & F \\
\hline Effets des traitements & 337,43 & 7 & 48,20 & 1,41 \\
A l'intérieur des groupes & 6484,30 & 189 & 34,17 & $\mathrm{p}=.203$ \\
Variation totale & 6821,73 & & & \\
\hline
\end{tabular}

\section{ANNEXE 3 : \\ CONSTRUCTION DE L'INDICE DE PROGRESSION IP}

Les élèves ont été soumis à un pré-test et un post-test en amont et en aval des séquences d'enseignement. Ces tests fournissent des scores sur 22 points. Lindice de progression permet de mesurer les acquisitions réalisées par les élèves de l'un à l'autre, au-delà de la seule différence de scores. Nous l'empruntons à Sarrazy (1996, 
Cf. p. 431-443). Les différentes étapes de la construction seront seulement résumées ici.

Pour chaque élève quatre types d'informations ont été distingués :

- P : nombre de progrès possibles (correspondant au nombre de problèmes échoués au pré-test);

- $\mathrm{p}_{\mathrm{o}}$ : nombre de progrès effectivement réalisés (nombre de problèmes échoués au pré-test et réussis au posttest);

- $\mathrm{R}$ : nombre de régressions possibles (correspondant au nombre de problèmes réussis au pré-test);

- $r_{\mathrm{o}}$ : nombre de régressions effectivement réalisées (nombre de problèmes réussis au pré-test et échoués au post-test).

Il est ainsi possible de déterminer : la fréquence des progressions $(\mathrm{Pp}=\mathrm{po} / \mathrm{P})$; et la fréquence des régressions $\left(P_{r}=r_{o} / R\right)$.

On pourrait penser à ce stade que la différence de fréquence de progression et de régression $\left(\mathrm{P}_{\mathrm{p}}-\mathrm{P}_{\mathrm{r}}\right)$ est satisfaisante pour rendre compte des acquisitions réalisées. Il n'en est rien. Lexemple fourni par Sarrazy est celui-ci : une fréquence de 0,50 ( $50 \%$ de progrès) peut être obtenue avec des scores très différents : $\mathrm{P}=2$ et $\mathrm{p}_{\mathrm{o}}=1 ; \mathrm{P}=8$ et $\mathrm{p}_{\mathrm{o}}=4 ; \mathrm{P}=16$ et $\mathrm{p}_{\mathrm{o}}=8 ;$ etc.

Il s'agit alors de pouvoir estimer, à un seuil de risque donné, la signification de l'écart existant entre les fréquences observées et un modèle théorique approprié, construit par Sarrazy. Nous en présenterons ici les principaux éléments.

\section{Calcul de la probabilité de réussir (ou d'échouer à) n problèmes sur $\mathbf{N}$}

La première question est la suivante : comment estimer la probabilité de réussir $n$ problèmes sur $N$ ?

En fonction de la probabilité de répondre au hasard compte tenu du type de réponse attendue aux problèmes posés $^{17}$, Sarrazy conclut qu'un élève a une chance sur deux de trouver la transformation attendue et une chance sur deux de réaliser la « bonne opération ». Il a donc une chance sur quatre de réussir et trois chances sur quatre d'échouer sur chaque problème en répondant au hasard. Ainsi, par exemple, à un élève ayant réalisé trois progrès sur cinq possibles, est associée la probabilité de réussite : $.25^{3} \times .75^{5-3}$.

Il faut alors multiplier cette probabilité associée à cet événement par le nombre de possibilités de réussir $n$ problèmes pris parmi $N$, soit par le nombre :

$$
C_{N}^{n}=\frac{n !}{n !(N-n) !}
$$

De cette manière, on obtient le calcul de la probabilité de réussir (ou d'échouer) à $n$ problèmes sur $N$ :

$$
\operatorname{Prob}(n, N)=C_{N}^{n} \times p^{n} \times(1-p)^{N-n}
$$

soit

$$
\operatorname{Prob}(n, N)=C_{N}^{n} \times .25^{n} \times .75^{N-n}
$$

Dans la formule ci-dessus :

$\mathrm{N}$ est le nombre de progrès possibles ( $\mathrm{P}$ ) ou de régressions possibles ( $\mathrm{R})$ au post-test ;

$n$ est le nombre de progrès observés $\left(\mathrm{p}_{\mathrm{o}}\right)$ ou de régressions observées $\left(\mathrm{r}_{\mathrm{o}}\right)$ au post-test ;

et $p$ est la probabilité de répondre correctement au hasard à un problème au post-test $(p=.25$; la probabilité de répondre faux est de $.75=1-\mathrm{p}$ ).

Qu'il s'agisse de progressions ou de régressions, il est à présent possible de choisir un seuil $S$ en deçà duquel on considère significatives les fréquences observées. Autrement dit, on admet que l'élève progresse (ou régresse) si la probabilité de réussir po problèmes sur $\mathrm{P}$ problèmes (respectivement d'échouer à ro problèmes sur R) est inférieure ou égale à un seuil $S$ choisi. Nous choisissons pour notre part un seuil $S=.20$. Si la probabilité est supérieure à $S$, il convient alors d'examiner la signification de cette probabilité de réussite ou d'échec.

\section{Estimation de la signification de la fréquence $n / N$}

Le problème est le suivant : lorsque la probabilité associée à l'événement $(n, N)$ est supérieure à $S$, nous pouvons affirmer que l'événement avait plus de $20 \%$ de chances de se réaliser. Même si ceci ne permet pas d'affirmer que l'élève a répondu au hasard, il convient d'évaluer la signification d'une telle fréquence.

Pour ce faire, on compare les fréquences observées au modèle théorique en posant l'hypothèse nulle : $H_{0}$ : 
l'élève ne progresse (régresse) pas. Dans ce cas $\mathrm{p}_{\mathrm{o}}=0\left(\mathrm{r}_{\mathrm{O}}=0\right)$; autrement dit, la fréquence des progrès (régressions) est nulle: $\mathrm{P}_{\mathrm{p}}=\mathrm{p}_{\mathrm{o}} / \mathrm{P}=0\left(\mathrm{P}_{\mathrm{r}}=\mathrm{r}_{\mathrm{o}} / \mathrm{R}=0\right)$.

Tab. c - Modèles théoriques des progrès et des régressions réalisés

\begin{tabular}{llllllll}
\hline & Progrès & & \multicolumn{3}{c}{ Régressions } \\
\cline { 2 - 6 } & Non réalisés & Observés & Total & Non réalisées & Observées & Total \\
\hline Contingence & $\mathrm{p}_{\mathrm{n}}$ & $\mathrm{p}_{\mathrm{o}}$ & $\mathrm{P}=\mathrm{p}_{\mathrm{n}}+\mathrm{p}_{\mathrm{o}}$ & Contingence & $\mathrm{r}_{\mathrm{n}}$ & $\mathrm{r}_{\mathrm{o}}$ & $\mathrm{R}=\mathrm{r}_{\mathrm{n}}$ \\
Modèle & $\mathrm{P}$ & 0 & $\mathrm{P}$ & Modèle & $\mathrm{R}$ & 0 & $\mathrm{r}$ \\
\hline
\end{tabular}

Il s'agit donc ici d'évaluer le risque que Pn (Pp ou Pr) soit nul ou plus petit que zéro avec la méthode de l'écartréduit :

$$
p_{p}-1,96 \sqrt{\frac{p_{p}\left(1-p_{p}\right)}{N}}>0 \quad \text { avec } N \times p_{p} \quad \text { et } \quad N \times\left(1-p_{p}\right)>5
$$

Si la valeur obtenue est positive, on pourra affirmer avec un risque d'erreur de $5 \%$ que la fréquence $\mathrm{P}_{\mathrm{n}}\left(\mathrm{P}_{\mathrm{p}}\right.$ ou $\mathrm{P}_{\mathrm{r}}$ ) est significativement différente de 0 ; en d'autres termes : l'élève a effectivement progressé ou régressé.

\section{Calcul de l'indice de progression}

Présentons maintenant le principe de calcul de l'indice de progression $\left(\mathrm{I}_{\mathrm{p}}\right)$ : il correspond à la différence observée entre la fréquence de progrès $\left(\mathrm{P}_{\mathrm{p}}\right)$ et la fréquence des régressions $\left(\mathrm{P}_{\mathrm{r}}\right)$ sous réserve toutefois que :

à chacune de ces fréquences soit associée une probabilité inférieure à $S$;

dans le cas où elles sont supérieures à S, elles devront être significativement différentes de 0 au seuil de $5 \%$. Au final, plusieurs cas sont à distinguer :

Tab. d - Conclusions de calcul de l'indice de progression

\begin{tabular}{|c|c|c|}
\hline Progrès $(\mathrm{Pp})$ & Régressions (Pr) & Indice de progression Ip \\
\hline - & - & $\begin{array}{l}\text { Ces élèves ont } 100 \% \text { de réussite au pré- } \\
\text { test et n'ont pas régressé au post-test. }\end{array}$ \\
\hline Prob $(p, P)>.20$ et $e, n . s .(1)$ & Prob $(r, R)>.20$ et e, n.s.(1) & Les élèves ne sont pas pris en compte. \\
\hline Prob $(p, P)<.20$ ou e, s.,. 05 & Prob $(r, R)>.20$ et e, n.s.(1) & $\mathrm{I}_{\mathrm{p}}=+\mathrm{p}_{\mathrm{p}}$ \\
\hline Prob $(p, P)>.20$ et e, n.s.(1) & Prob $(r, R)<.20$ ou e, s.,. 05 & $\mathrm{I}_{\mathrm{p}}=-\mathrm{p}_{\mathrm{r}}$ \\
\hline Prob $(p, P)<.20$ ou e, s.,. 05 & Prob $(r, R)<.20$ ou e, s.,. 05 & $\mathrm{I}_{\mathrm{p}}=\mathrm{p}_{\mathrm{p}}-\mathrm{p}_{\mathrm{r}}$ \\
\hline
\end{tabular}

(1) ou bien e non calculable.

Sur 197 élèves, 4 ont eu $100 \%$ de réussite au pré-test et n'ont pas régressé au post-test ( $1^{\text {re }}$ ligne du tableau), et l'indice de progression n'est pas calculable pour 17 ( $2^{\mathrm{e}}$ ligne du tableau). Ainsi, l'indice $\mathrm{I}_{\mathrm{p}}$ est calculable pour 176 élèves soient $89 \%$ de l'échantillon initial. 
1. Nous entendons « idéologie " au sens de ce que disent les professeurs à propos de ce qu'ils pensent qu'il est « bon » de faire sans référence à une justification autre que leur croyance; en cela, le sens est fort proche de la conception althussérienne de l'idéologie.

2. Les interactions maître-élèves(s) peuvent en effet être considérées comme des objets d'étude centraux des recherches en éducation, depuis les travaux princeps de Flanders (1966), Mc Dermott (1976), Bayer (1973, 1979), Crahay (1989), Altet (1994)... À ce jour, les publications qui leur sont consacrées continuent d'être nombreuses (cf. par exemple, Sarrazy (2001), Guernier, Durand-Guerrier \& Sautot (2007), Filliettaz \& Schubauer-Leoni (2008), etc.).

3. Dans ce jeu, les actions permettant d'aménager le milieu (lato sensu, i.e. au sens de la théorie des situations, Brousseau, 1998) et, plus spécifiquement, les interactions verbales, sont les seuls instruments disponibles pour le professeur : interroger un bon élève lui permettra de sortir d'une impasse, d'accélérer la leçon sous la pression du temps de l'horloge, rectifier publiquement l'erreur d'un plus faible ou au contraire ne pas lui répondre aura d'importants effets sur l'avancée du temps didactique, etc. 4. Les interactions maître-élève(s) sont en effet fréquemment examinées sous deux aspects principaux que nous laisserons de côté dans cette analyse : d'une part en fonction de ce qu'elles "révèlent" d'un style professoral particulier (Postic, 1971 ; Bressoux, 1990) ; d'autre part sous l'angle de leur étroite correspondance avec des caractéristiques non didactiques (socioculturelles, sexuées, etc.) des publics scolaires (e.g., Felouzis, 1993). Ici, c'est à leur fonctionnalité pleinement didactique que nous nous intéresserons, dans la perspective de travaux tels que ceux de Giroux et René De Cotret (2003) par exemple, qui les articulent d'ailleurs avec la question du temps didactique. 5. Pour Jakobson, dans une interaction sociale, un énoncé est " phatique " dès lors qu'il vise à maintenir la communication et à vérifier que le canal de communication fonctionne bien (par exemple : «Attends un peu », « On verra cela plus tard $» . .$.$) .$

6. Pour une présentation rapide des 6 structures additives de la typologie de Vergnaud, cf. annexe 1.

7. Comme le soulignait en particulier Vergnaud, l'utilisation des problèmes TTT dans le domaine arithmétique « permettrait de donner du sens à l'idée de solution négative (cette idée est contre intuitive pour la plupart des élèves, car ceux-ci se représentent les nombres comme des quantités ou des grandeurs, de ce fait positives), mais également de montrer les différents raccourcis que représente le raisonnement algébrique. » (1990b, p. 81).

8. Pour plus de précisions sur la construction des niveaux scolaires, cf. annexe 2.

9. On trouvera leur présentation détaillée dans Chopin (2007).

10. Parmi ces dernières, on trouve par exemple des interactions visant à maintenir l'ordre social (e.g. des réprimandes), des interactions instrumentales ou de gestion (e.g. à propos du matériel nécessaire, de l'organisation de la journée, etc.), et enfin des interactions dites « d'accompagnement » (plutôt tournées vers le soutien affectif de l'élève).

11. Ce qui ne signifie pas qu'elle soit erronée. Une réponse juste survenue trop tôt peut par exemple perturber la progression didactique d'un enseignant.

12. Pour les bons élèves $(r=.80, \mathrm{p} .<.02)$; pour les moyens $(\mathrm{r}=.58, \mathrm{p} .<.15)$; pour les faibles $(\mathrm{r}=.67, \mathrm{p} .<.10)$.

13. Une analyse de segmentation avait été réalisée pour établir cela (cf. Chopin, 2007).

14. Le codage en classes a été réalisé via le logiciel Statbox. Nous avons choisi le regroupement en classes optimales, c'est-à-dire où le calcul des classes est basé sur la minimisation de l'inertie intra-classe (les classes sont donc les plus compactes possibles). Statbox utilise l'algorithme de Anderberg (algorithme d'amélioration itérative d'une solution initiale).

15. Nous avions relevé l'avis des professeurs sur le niveau scolaire de leurs élèves de manière à nous assurer de la pertinence de notre mesure. Les recherches en éducation ont en effet largement établi l'influence de la perception que se font les professeurs de leurs élèves (et de leur niveau scolaire) sur leurs comportements d'enseignement (Rosenthal \& Jacobson, 1997 ; Bressoux \& Pansu, 2003). Aussi était-il important de construire une variable niveau scolaire qui puisse tout à la fois garantir une objectivité de la mesure sur l'ensemble de l'échantillon (nous baser sur les seules déclarations des professeurs n'aurait pas garanti l'homogénéité de la signification de la variable niveau scolaire entre les huit classes), et de vérifier le lien entre ces deux définitions du niveau scolaire.

16. La normalité des distributions et l'homogénéité des variances ont été vérifiées.

17. En effet, dans toutes les classes observées par Sarrazy, comme dans celles que nous avons nous-mêmes observées, les élèves savent, à l'issue des leçons qu'ils ont reçues, que la réponse qu'ils doivent fournir est de la forme " gagner/ perdu $\mathrm{x}$ billes » et que le $\mathrm{x}$ en question s'obtient par une soustraction ou par une addition. 